\title{
Associations between properties linked with persistence in a collection of Staphylococcus aureus isolates from bovine mastitis
}

\author{
Marjorie Bardiau $^{\mathrm{a}, *}$, Johann Detilleux ${ }^{\mathrm{b}}$, Frédéric Farnir ${ }^{\mathrm{c}}$, Jacques G. Mainil ${ }^{\mathrm{a}}$, \\ Isabelle Ote ${ }^{\mathrm{a}, 1}$ \\ a Bacteriology, Department of Infectious Diseases, Faculty of Veterinary Medicine, University of Liège, Sart-Tilman, Bât. $43 a$, B-4000 Liège, \\ Belgium \\ ${ }^{\mathrm{b}}$ Quantitative Genetics Group, Department of Animal Production, Faculty of Veterinary Medicine, University of Liège, Sart-Tilman, Bât. \\ 43a, B-4000 Liège, Belgium \\ ${ }^{\mathrm{c}}$ Biostatistics, Bioinformatics and Animal Selection, Department of Animal Production, Faculty of Veterinary Medicine, University of Liège, \\ Sart-Tilman, Bât. 43a, B-4000 Liège, Belgium
}

\section{A R T I C L E I N F O}

Article history:

Received 6 May 2013

Received in revised form 3 December 2013

Accepted 13 December 2013

\section{Keywords:}

S. aureus

Bovine mastitis

Biofilm

Capsule

Intracellular survival

Persistence

\begin{abstract}
A B S T R A C T
Staphylococcus aureus is recognized worldwide as a pathogen causing many serious diseases in humans and animals, and is one of the most important etiological agents of clinical and subclinical bovine mastitis. The aim of the present study was to investigate and correlate properties, that may be associated with persistent mastitis, of $S$. aureus strains isolated from milk of cows suffering from mastitis: (i) expression of capsular antigens (CP5 or CP8) by specific ELISA; (ii) intracellular survival by invasion of MAC-T cells; and (iii) biofilm production by spectrophotometry analysis after growth in TSB glc. $_{\text {. }}$ The results showed that (i) the proportion of strains expressing capsular antigen was higher in cap8- than in cap5-positive isolates; (ii) a correlation was observed between the capsular profile and the intracellular survival as well as the biofilm production; and (iii) the capsular profile, biofilm production and intracellular survival were associated with only two agr-groups. Statistical and clustering analysis allowed us to establish different profiles that could be associated with in vivo persistence. Indeed, isolates belonging to agr group II, expressing the capsular antigen CP8 and showing low intracellular survival are probably better adapted to an extracellular niche. Conversely, isolates belonging to agr group I that do not express any capsular antigen (CP5 or CP8) but show high intracellular survival are probably better adapted to an intracellular niche.
\end{abstract}

(c) 2013 Elsevier B.V. All rights reserved.

\section{Introduction}

Staphylococcus aureus ( $S$. aureus) is one of the most common aetiological agents of contagious bovine mastitis (Watts, 1988). Although S. aureus strains can cause

\footnotetext{
* Corresponding author. Tel.: +32 436695 22; fax: +32 43664261 . E-mail addresses: mbardiau@ulg.ac.be, mbardiau@gmail.com (M. Bardiau).

1 Current address: Coris BioConcept, Science Park CREALYS, rue Jean Sonet 4a, B-5032 Gembloux, Belgium.
}

acute and clinical mastitis with macroscopic alteration of the milk, such bacterial infections can evolve towards chronic and subclinical mastitis, without macroscopic alteration of the milk but with high cell counts and persistence of the bacteria in the mammary gland. Staphylococcal subclinical mastitis account for up to $30 \%$ of all cases of bovine mastitis (Halasa et al., 2007). They represent a serious economical problem for dairy farmers with reduction of milk production and quality, prolonged antibiotic treatments and premature culling. The identification of the staphylococcal properties 
associated with long-lasting infections is therefore of the upmost importance.

For instance, biofilm formation impairs the action of both the host immune system and the antimicrobial agents, and therefore represents one of the most important survival mechanisms of bacteria persistently colonizing the extracellular niche (Costerton et al., 1999; Melchior et al., 2006). Nevertheless, several factors, environmental as well as genetic, influence biofilm formation by $S$. aureus.

$S$. aureus is also known to penetrate, survive, and even multiply within a large variety of eukaryotic cells, such as the epithelial cells of the mammary gland or the immune cells (Almeida et al., 1996; Kerro Dego et al., 2002). This survival within the intracellular niche protects the bacteria from the antibiotics commonly used in mastitis treatment, but also enables them to persist in the host for a long time without causing apparent inflammation (Boulanger et al., 2003; Garzoni and Kelley, 2009).

The role of the staphylococcal capsule polysaccharides (CP) in this internalization has been described. More precisely, the absence of $\mathrm{CP}$ expression was shown to enhance adherence (Pohlmann-Dietze et al., 2000) and invasion (Tuchscherr et al., 2005) of endothelial and epithelial cells by $S$. aureus. Furthermore, capsule-negative $S$. aureus bacteria were shown to induce chronic experimental mastitis in mice, suggesting that the loss of capsule expression may enhance the persistence of $S$. aureus in the mammary glands (Tuchscherr et al., 2005).

The aim of the present work was thus to determine whether association of specific in vitro tests could establish a profile of in vivo persistence. We therefore investigated the correlation of features described to be associated with longlasting infections, respectively capsular antigen identity and expression, biofilm formation, and intracellular survival, on a collection of $S$. aureus strains isolated from cases of bovine mastitis in Belgium. These phenotypic features were also supplemented by phylogenetic analyses, such as agr-typing and PFGE. Statistical and clustering analyses were performed to associate the different tests together and to evaluate the homogeneity and the distribution of profiles.

\section{Materials and methods}

\subsection{Bacterial isolates}

A total of 229 bovine $S$. aureus isolates, collected between 2005 and 2008 from bovine mastitis cases in different areas of Wallonia (southern part of Belgium), were assayed in this study. All isolates have already been virulotyped (Ote et al., 2011). Two reference strains from bovine mastitis were included in this collection: $S$. aureus ATCC 29740 (N305) and S. aureus ATCC 31885 (NL6).

\subsection{Capsular serotyping}

The prevalence of the capsule-encoding genes (cap5 and cap8) among the isolates of the collection has already been determined by PCR and are shown in Table 1 (Ote et al., 2011). However, capsular serotyping was performed using ELISA test through the detection of capsular polysaccharides types 5 and 8 (CP5 and CP8) from bacteria grown on Columbia agar. Briefly, strains were cultured from frozen stocks onto Columbia sheep blood agar plates and incubated at $37^{\circ} \mathrm{C}$ overnight. Bacteria were then collected from agar plates by washing the plates with sterile PBS and adjusted to $10^{10} \mathrm{CFU} / \mathrm{ml}$ by optical density (OD) measure. The capsular serotype of $10^{9} \mathrm{CFU}$ of each strain was determined by enzyme-linked immunosorbent assay (ELISA) in 96-well microplates, using specific monoclonal and polyclonal antibodies (kindly provided by GSK Biologicals, Belgium) and horseradish peroxidase-based quantification. The OD of each well was measured at $490 \mathrm{~nm}$ using a microplate reader (Bio-Rad). The results were collected from at least two independent experiments in which the capsule production of each tested culture was evaluated in triplicates. OD values were compared to those obtained with $S$. aureus CP reference strains, respectively the CP5-positive strain Lowenstein (ATCC 49521) and CP8positive strain Wright (ATCC 49525), and isolates that tested negative for CP5- and CP8- ELISA were defined as non-typeable (NT).

Table 1

Capsular profile, intracellular survival, biofilm formation and agr-groups frequency distribution.

\begin{tabular}{|c|c|c|c|c|c|c|c|c|c|c|c|}
\hline & & \multicolumn{3}{|c|}{$\begin{array}{c}\text { Intracellular } \\
\text { survival }\end{array}$} & \multicolumn{3}{|c|}{ Biofilm formation } & \multicolumn{4}{|c|}{ agr-groups } \\
\hline & & $<2 \%$ & $>2 \%$ & No & Weak & Moderate & Strong & $\mathbf{I}$ & II & III & IV \\
\hline \multirow{10}{*}{$\begin{array}{l}\text { Capsular } \\
\text { profile }\end{array}$} & cap $5 / \mathrm{NT}$ & 13 & 32 & 6 & 17 & 12 & 10 & 39 & 4 & 2 & 0 \\
\hline & cap5/CP5 & 5 & 23 & 4 & 13 & 7 & 4 & 21 & 7 & 0 & 0 \\
\hline & $\operatorname{cap} 8 / \mathbf{N T}$ & 16 & 20 & 1 & 12 & 12 & 11 & 33 & 2 & 1 & 0 \\
\hline & cap8/CP8 & 95 & 25 & 29 & 63 & 20 & 8 & 72 & 46 & 1 & 1 \\
\hline & \multirow{2}{*}{\multicolumn{2}{|c|}{$\begin{array}{c}\text { Intracellular } \\
\text { survival }\end{array}$}} & $<2 \%$ & 33 & 64 & 21 & 11 & 80 & 46 & 2 & 1 \\
\hline & & & $>2 \%$ & 7 & 41 & 30 & 22 & 85 & 13 & 2 & 0 \\
\hline & & & & & \multirow{4}{*}{\multicolumn{2}{|c|}{$\begin{array}{l}\text { Biofilm } \\
\text { formation }\end{array}$}} & No & 18 & 20 & 2 & 0 \\
\hline & & & & & & & Weak & 76 & 28 & 1 & 0 \\
\hline & & & & & & & Moderate & 43 & 7 & 0 & 1 \\
\hline & & & & & & & Strong & 28 & 4 & 1 & 0 \\
\hline
\end{tabular}

Significant correlations $(P<0.0005)$ are highlighted in bold. cap, capsule-encoding gene; CP, capsular polysaccharide; NT: non-typeable. Intracellular survival is presented as the \% of the initial inoculum. agr-typing: I, II, III, IV, agr-groups. 


\subsection{Invasion assay}

Bovine mammary epithelial cells (MAC-T) were used for in vitro bacterial internalization assays as previously described (Boulanger et al., 2007; Brouillette et al., 2003; Buzzola et al., 2007; Tuchscherr et al., 2005). MAC-T cells were grown in Dulbecco's modified Eagle's medium (DMEM), supplemented with 10\% heat-inactivated foetal bovine serum (FBS), non-essential amino acids, insulin and antibiotics. Prior to each experiment, MAC-T cells were seeded at $\sim 6 \times 10^{4}$ cells/well in 24-well culture plates and incubated for 3 days at $37{ }^{\circ} \mathrm{C}$ in $5 \% \mathrm{CO}_{2}$. Approximately $16 \mathrm{~h}$ prior to the invasion experiments, cells were washed twice with PBS and incubated with invasion medium (growth medium without antibiotics containing only $1 \%$ heat-inactivated FBS). Concurrently, $S$. aureus strains were incubated in Brain-Heart Infusion (BHI) broth overnight with shaking at $37^{\circ} \mathrm{C}$. Bacteria concentration was then adjusted to $10^{9} \mathrm{CFU} / \mathrm{ml}$ in fresh BHI by optical density (OD) measure, and confluent MAC-T cell monolayers $\left(\sim 2.5 \times 10^{5}\right.$ cells/well $)$ were inoculated with $10^{7} \mathrm{CFU}$ of $S$. aureus $(10 \mu \mathrm{l} /$ well, multiplicity of infection $\sim 40$ ) and incubated at $37{ }^{\circ} \mathrm{C}$ in $5 \% \mathrm{CO}_{2}$. After $1 \mathrm{~h}$, supernatants of the co-cultures were removed and cells were washed twice with PBS to remove unattached bacteria. One $\mathrm{ml}$ of invasion medium supplemented with lysostaphin $(20 \mu \mathrm{g} / \mathrm{ml})$ was added to each well to lyse extracellular bacteria, and incubation was prolonged for an additional $2 \mathrm{~h}$ at $37{ }^{\circ} \mathrm{C}$ in $5 \% \mathrm{CO}_{2}$. Supernatants were then collected and plated on Columbia sheep blood agar plates to verify the lysis of extracellular bacteria by lysostaphin. After two PBS washing steps, the MAC-T cells were detached by incubation for $15 \mathrm{~min}$ at $37^{\circ} \mathrm{C}$ with $100 \mu \mathrm{l} /$ well of trypsin and then lysed to release intracellular staphylococci by addition of $900 \mu \mathrm{l} /$ well of sterile distilled water containing $0.025 \%$ Triton X-100. The cell lysates were carefully suspended, serially diluted, and plated on Columbia sheep blood agar plates to quantify CFU number after overnight growth at $37^{\circ} \mathrm{C}$. Results are expressed as a percentage of recovered bacteria from the initial inoculum.

\subsection{Biofilm production}

Biofilm formation was evaluated by spectrophotometry in microplates using safranin staining. Briefly, strains were incubated in Tryptic Soy Broth (TSB) overnight with shaking at $37^{\circ} \mathrm{C}$. Cultures were then diluted $1: 100$ in TSB containing $0.25 \%$ glucose ( $\mathrm{TSB}_{\mathrm{glc}}$ ) (Melchior et al., 2009) and transferred into wells of sterile 96-well polystyrene tissue culture (TC) plates. $\mathrm{TSB}_{\mathrm{glc}}$ without bacteria served as negative control. The TC plates were incubated at $37^{\circ} \mathrm{C}$ for $24 \mathrm{~h}$. The supernatant was then discarded and the wells were carefully washed twice with sterile PBS. The plates were dried, stained with safranin $0.1 \%(\mathrm{w} / \mathrm{v})$ for $10 \mathrm{~min}$, further washed twice with distilled water and dried again at $37^{\circ} \mathrm{C}$. A mixture of $50 \%$ ethanol and $50 \%$ acetic acid was added to each well and plates were incubated at room temperature for $15 \mathrm{~min}$. Finally, the OD of each well was measured at $490 \mathrm{~nm}$ using a microplate reader (Bio-Rad). The results were collected from at least two independent experiments in which the biofilm formation of each culture tested was evaluated in triplicates. The quantitative classification of biofilm production was based on cut-off value (ODc) and average OD values (Stepanovic et al., 2007), leading to four categories of strains: $\mathrm{OD} \leq \mathrm{ODC}=$ no biofilm producer; $\mathrm{ODc}<\mathrm{OD} \leq 2 \times \mathrm{ODc}=$ weak biofilm producer; $2 \times$ ODc $\times$ ! $\mathrm{OD} \leq 4 \times$ ODc $=$ moderate biofilm producer; $4 \times$ ODc $<$ $\mathrm{OD}=$ strong biofilm producer.

\section{5. agr-typing}

agr-groups were determined by multiplex PCR as previously described (Gilot et al., 2002). In brief, multiplex PCRs were performed on $2 \mu$ l of DNA using Taq DNA polymerase (New England BioLabs) and $1 \mu \mathrm{M}$ of each of the following primers: Pan (5'-ATG CAC ATG GTG CAC ATG C-3'), agr1 (5'-GTC ACA AGT ACT ATA AGC TGC GAT), agr2 (5'-TAT TAC TAA TTG AAA AGT GGC CAT AGC-3'), agr3 (5'GTA ATG TAA TAG CTT GTA TAA TAA TAC CCA G-3'), and agr4 (5'-CGA TAA TGC CGT AAT ACC CG-3'). Amplifications were performed with the following PCR programme: 1 cycle at $94{ }^{\circ} \mathrm{C}$ for $1 \mathrm{~min} ; 26$ cycles at $94{ }^{\circ} \mathrm{C}$ for $30 \mathrm{~s}, 55^{\circ} \mathrm{C}$ for $30 \mathrm{~s}$, and $72{ }^{\circ} \mathrm{C}$ for $1 \mathrm{~min}$; and finally 1 cycle at $72^{\circ} \mathrm{C}$ for $10 \mathrm{~min}$. All PCR products were separated by electrophoresis in $1.5 \%(\mathrm{w} / \mathrm{v})$ agarose gel. Gels were stained with ethidium bromide and photographed under UV light. Patterns were compared to those obtained with S. aureus agr group reference strains, respectively the N305 strain (ATCC 29740) for agr group I, the Mu50 strain (ATCC 700699) for agr group II, the Seattle 1945 strain (ATCC 25923) for agr group III, and the A880740 strain for agr group IV.

\subsection{Pulsed field gel electrophoresis}

Pulsed field gel electrophoresis (PFGE) analyses were performed on all strains. In brief, lysostaphin pre-treated bacterial cells were embedded in $1.8 \%$ certified low-melt agarose (Bio-Rad), lysed in a $0.5 \mathrm{M}$ EDTA ( $\mathrm{pH} 8$ ) buffer containing $10 \% \mathrm{~N}$-lauroylsarcosine and $2 \mathrm{mg} / \mathrm{ml}$ lysozyme, and treated with $1 \mathrm{mg} / \mathrm{ml}$ proteinase $\mathrm{K}$. The genomic DNA was digested with $20 \mathrm{U}$ of SmaI (Sigma) according to the manufacturer's instructions. The restricted fragments were separated through a $1 \%$ pulsed-field certified agarose (Bio-Rad) gel in 0.5\% TBE buffer by using a CHEF MAPPER (Bio-Rad), at $6.0 \mathrm{~V} / \mathrm{cm}$ for $21 \mathrm{~h}$, with pulsed times ranging from 5 to $60 \mathrm{~s}$, with an angle of $120^{\circ}$ and a linear ramp factor. The size of each DNA band was estimated by Biogene (Vilber Lourmat).

\subsection{Statistical analysis}

For calculation of the statistical significance of the observed frequency distributions, contingency tables of the random expected values were determined and Chisquare tests were performed. $P$ values lower than 0.05 were considered significant. K-means clustering method, adapted for the clustering of categorical data, was used to group similar strains into homogeneous groups (procedure fastclus, sas 9.1). 


\section{Results}

\subsection{Capsular serotyping}

Seventy-three strains (31.9\%) harboured the cap5 gene while 156 (68.1\%) tested positive for the cap8 gene (Table 1) (Ote et al., 2011). Here, we further assessed the effective production of the CP5 and CP8 polysaccharides by ELISA analysis. Twenty-eight of the 229 S. aureus isolates (12.2\%) expressed CP5, 120 (52.4\%) expressed CP8, and $81(35.4 \%)$ were defined as non-typeable (Table 1$)$.

\subsection{Invasion assay}

We observed a high variability in the percentage of recovered bacteria from the initial inoculum $2 \mathrm{~h}$ postinternalization (from 0 to 95\%) into MAC-T cells. Nevertheless, our data highlighted three populations of strains, corresponding to strains showing distinct survival rates, respectively (i) $0.5 \pm 0.5 \%$, (ii) $6.7 \pm 3.9 \%$ and (iii) $50.6 \pm 29 \%$ of the initial inoculum. However, after further analysis (see contingency tables below), we observed that populations (ii) and (iii) behaved similarly in all other features. In order to simplify the results presentation, we thus chose to keep the distribution of the strains of our collection into two populations. We determined that 129 (56.3\%) showed an invasion rate lower than $2 \%$ of the initial inoculum, while 100 (43.7\%) showed an invasion rate greater than $2 \%$ (Table 1 ).

\subsection{Biofilm production}

In $\mathrm{TSB}_{\mathrm{glc}}$, only 40 strains (17.5\%) did not produce any biofilm, while the majority were biofilm producers, respectively weak $(n=105 ; 45.8 \%)$, moderate $(n=51$; $22.3 \%)$ or strong $(n=33 ; 14.4 \%)$ producers (Table 1$)$.

\section{4. agr-typing and PFGE}

In our collection, all four agr-groups were detected. The majority of the isolates ( $n=165 ; 72.1 \%$ ) belonged to $a g r$ group I, 59 (25.8\%) belonged to agr group II, 4 (1.7\%) belonged to agr group III and only one strain $(0.4 \%)$ belonged to agr group IV (Table 1).

Concerning PFGE results, the 229 strains showed 157 distinct pulsotypes (data not shown). The four main pulsotypes, namely A, B, C and D, grouped 33 (14.4\%), $28(12.2 \%), 7(3.1 \%)$ and $8(3.5 \%)$ isolates respectively (Table 1). All the other pulsotypes were typically represented by only one strain ( $n=153 ; 66.8 \%$ ). These results confirm the heterogeneity of our collection, information quite important here regarding the lack of anamnesis and farm origin of our strains.

\subsection{Statistical associations}

In order to determine whether the studied features could be correlated, we disposed their frequency distributions in contingency tables, and the observed values were compared to the random expected values by Chi-square tests. $P$ values lower than 0.05 were considered significant. The observed frequencies that were statistically higher than randomly expected were highlighted in bold in all contingency tables.

We first observed that more cap8-positive strains express CP8, whereas more cap5-positive strains are defined as non-typeable (Table $1, P<0.0005$ ), suggesting that CP8 strains tend to be capsulated, while CP5 strains tend to remain acapsulated.

We then observed that more cap8/CP8-strains show a low invasion rate $(<2 \%)$, whereas more NT-strains show a high invasion rate $(>2 \%)$ (Table $1, P<0.0005)$. Similarly, more cap8/CP8-strains do not produce, or weakly, biofilm in $\mathrm{TSB}_{\mathrm{glc}}$, whereas more NT-strains are moderate or strong producers (Table $1, P<0.0005$ ).

We also observed that more strains showing a low invasion rate do not produce, or weakly, biofilm in $\mathrm{TSB}_{\mathrm{glc}}$, and that more strains showing a high invasion rate are moderate or strong biofilm producers in $\mathrm{TSB}_{\text {glc }}$ (Table 1 , $P<0.0005$ ).

Furthermore, we observed that more cap8/CP8 strains belong to agr group II, while more NT-strains belong to agr group I (Table $1, P<0.005)$. Concerning biofilm production, more agr group I strains produce biofilm in $\mathrm{TSB}_{\text {glc }}$, while more agr group II strains do not produce biofilm in $\mathrm{TSB}_{\mathrm{glc}}$ (Table $1, P<0.0005)$. Finally, we found that more agr group II strains show a low invasion rate $(<2 \%)$, whereas more agr group I strains show a high invasion rate $(>2 \%)$ (Table 1 , $P<0.0005$ )

We then divided the strains into eight groups according to their capsular profile and intracellular survival ability, and we compared their PFGE pulsotypes (Table 2). Strains from pulsotypes $C$ and D were shown to be NT-strains exclusively, either cap8- or cap5-positive respectively. Conversely, most of the strains from pulsotypes A and B were CP8-strains showing a low rate of internalization $(<2 \%)$. Interestingly, $55.8 \%(n=53)$ of this subgroup of strains (cap8/CP8, <2\%) were only represented by these two pulsotypes $A$ and $B$, suggesting that these strains may be more clonal than all other groups.

\subsection{Clustering method (Table 3)}

The cluster 1 grouped strains showing a low invasion rate in MAC-T cells and belonging to agr group I. The cluster

Table 2

Distribution of the strains according to their capsular profile, intracellular survival and PFGE pulsotypes.

\begin{tabular}{llrrrrrr}
\hline \multirow{2}{*}{$\begin{array}{l}\text { Capsular } \\
\text { profile }\end{array}$} & $\begin{array}{l}\text { Intracellular } \\
\text { survival }\end{array}$ & \multicolumn{6}{c}{ PFGE typing } \\
\cline { 3 - 6 } & & \multicolumn{1}{c}{ A } & \multicolumn{1}{c}{ B } & C & D & Other & \\
\hline cap5/NT & $<2 \%$ & 0 & 0 & 0 & 1 & 12 & 13 \\
& $>2 \%$ & 0 & 0 & 0 & 7 & 25 & 32 \\
cap5/CP5 & $<2 \%$ & 0 & 0 & 0 & 0 & 5 & 5 \\
& $>2 \%$ & 0 & 0 & 0 & 0 & 23 & 23 \\
cap8/NT & $<2 \%$ & 0 & 2 & 3 & 0 & 11 & 16 \\
& $>2 \%$ & 2 & 0 & 4 & 0 & 14 & 20 \\
cap8/CP8 & $<2 \%$ & 27 & 26 & 0 & 0 & 42 & 95 \\
& $>2 \%$ & 4 & 0 & 0 & 0 & 21 & 25 \\
& & 33 & 28 & 7 & 8 & 153 & 229 \\
\hline
\end{tabular}

Capsular profile: cap, capsule-encoding gene; $\mathrm{CP}$, capsular polysaccharide; NT, non-typeable. Intracellular survival: presented as the \% of the initial inoculums. PFGE typing: A, B, C, D, the four main pulsotypes. 
Table 3

Clustering of the strains according to their capsular profile, intracellular survival, biofilm formation and agr-groups (K-Means method).

\begin{tabular}{llrrrrr}
\hline & & \multicolumn{2}{c}{ Clusters } & & & \\
\cline { 2 - 6 } & & 1 & 2 & 3 & 4 & 5 \\
\hline Capsular profile & cap5/NT & 10 & $\mathbf{2 3}$ & 5 & 4 & 3 \\
& cap5/CP5 & 2 & 8 & 6 & 9 & 3 \\
& cap8/NT & 10 & 7 & 12 & 1 & 6 \\
& cap8/CP8 & 21 & 9 & 14 & 2 & $\mathbf{7 4}$ \\
Intracellular survival & $<2 \%$ & $\mathbf{4 3}$ & 0 & 0 & 0 & $\mathbf{8 6}$ \\
& $>2 \%$ & 0 & $\mathbf{4 7}$ & $\mathbf{3 7}$ & $\mathbf{1 6}$ & 0 \\
Biofilm formation & No & 14 & 3 & 1 & 1 & $\mathbf{1 9}$ \\
& Weak & 0 & $\mathbf{3 7}$ & 4 & 4 & $\mathbf{6 4}$ \\
& Moderate & 19 & 0 & 5 & 5 & 2 \\
& Strong & 10 & 7 & 6 & 6 & 1 \\
& I & $\mathbf{4 0}$ & $\mathbf{4 6}$ & $\mathbf{3 7}$ & 2 & 40 \\
& II & 0 & 0 & 0 & $\mathbf{1 3}$ & $\mathbf{4 6}$ \\
& III & 2 & 1 & 0 & 1 & 0 \\
& IV & 1 & 0 & 0 & 0 & 0 \\
& Total & $\mathbf{4 3}$ & $\mathbf{4 7}$ & $\mathbf{3 7}$ & $\mathbf{1 6}$ & $\mathbf{8 6}$ \\
\hline
\end{tabular}

Significant correlations $(P<0.0001)$ are highlighted in bold. Capsular profile: cap, capsule-encoding gene; $\mathrm{CP}$, capsular polysaccharide; NT, non-typeable. Intracellular survival: presented as the \% of the initial inoculums. agr-typing: I, II, III, IV, agr-groups.

2 grouped strains that were cap5-positive but tested negative in vitro for CP5 ELISA, showed a high invasion rate in MAC-T cells, formed weak biofilm in $\mathrm{TSB}_{\text {glc }}$ and belonged to agr group I. The cluster 3 grouped strains showing a high invasion rate in MAC-T cells and belonging to agr group I. The cluster 4 grouped strains showing a high invasion rate in MAC-T cells and belonging to agr group II. The cluster 5 grouped strains that were cap8-positive and tested positive in vitro for CP8 ELISA, showed a low invasion rate in MAC-T cells, formed weak or no biofilm in TSB $_{\text {glc }}$ and belonged to agr group II. Statistical significances were included in the Table 3 and highlighted in bold $(p<0.0001)$

\section{Discussion}

Although different properties associated with the persistence of $S$. aureus in the host, especially the mammary gland, have already been individually, or by pair, explored, to our knowledge, this is the first study correlating all features at the same time on such a large collection of strains.

Although here presented as \% of the initial inoculum, the invasion rate values that we observed correspond to those described in the literature (Boulanger et al., 2007; Brouillette et al., 2003; Buzzola et al., 2007; Tuchscherr et al., 2005). Thus, in accordance with the observation that the presence of a capsule impedes cellular invasion (Buzzola et al., 2007; Tuchscherr et al., 2005), we confirmed that capsulated strains are less able than acapsulated to invade and survive intracellularly. However, the fact that some capsulated isolates still show internalization capacity further suggests that the absence of capsule is not the only property facilitating invasion of eukaryotic cells (for review, Tuchscherr et al., 2010).
We also correlated biofilm production to both the capsule profile and the intracellular survival. Thus, NTstrains or strains with an intracellular survival rate higher than $2 \%$ were shown to be biofilm producers in $\mathrm{TSB}_{\text {glc }}$. Inversely, more cap8/CP8-strains than expected or strains with an intracellular survival rate lower than $2 \%$ do not produce biofilm in $\mathrm{TSB}_{\text {glc }}$.

It should be noted that, although our statistical analysis suggests a good correlation between the studied features, even if performed in different media, our results could be strengthened by analysis of bacteria grown in similar conditions. It is besides quite intuitive to consider that milk serum used in vitro reflects more closely the situation encountered in vivo during mastitis. Further investigations on capsular profile, intracellular survival and biofilm formation will therefore be conducted on bacteria grown in milk serum, or in classical medium supplemented with different milk compounds, considering the potential influence of these compounds on genes expression. Such influence on biofilm production has already been demonstrated for S. aureus (Melchior et al., 2009) but also for other bacterial species, i.e. Streptococcus uberis (Mitchell et al., 2010; Varhimo et al., 2011). Particularly, Melchior et al. have shown that the growth media influences biofilm formation. Indeed, they obtained different biofilm production results in $\mathrm{TSB}_{\text {glc }}$ and in milk serum. Moreover, they observed that biofilm formation was higher for agr group I strains than agr group II strains in $\mathrm{TSB}_{\mathrm{glc}}$, but lower for $a g r$ group I strains than agr group II strains in milk serum. Their results in $\mathrm{TSB}_{\mathrm{glc}}$ are in agreement with our results. Thus, the study of biofilm formation in milk serum for our collection of strains would be particularly interesting.

In accordance with the literature (Boulanger et al., 2007; Brouillette et al., 2003; Buzzola et al., 2007; Tuchscherr et al., 2005), we further observed that more agr group I strains show a high invasion rate $(>2 \%)$, while more agr group II strains show a low invasion rate $(<2 \%)$. In parallel, we also highlighted that more agr group I strains remain acapsulated (NT-strains), whereas more agr group II strains express CP8. However, as we did not assess the functionality of the agr system of all strains of the collection, we cannot determine whether the absence of $\mathrm{CP}$ expression or the high invasion rate are directly linked to regulation by the agr locus, or whether other factors, such as those related to the culture medium used, could play a role. In accordance with the literature, strains belonging to agr group I produce biofilm in $\mathrm{TSB}_{\mathrm{glc}}$, while conversely, more strains than expected belonging to $a g r$ group II are non-producers in $\mathrm{TSB}_{\text {glc }}$.

Based on all these in vitro studied features, the clustering of the strains allowed us to establish different profiles that could be associated with specific niches (intracellular or extracellular). Thus, we can assume that cap5-positive strains belonging to agr group I, which in vitro test negative for CP5 ELISA and show a high invasion rate in MAC-T cells (cluster 2), could correspond to strains adapted to the intracellular niche. Conversely, cap8positive strains belonging to agr group II, which express CP8 in vitro and show a low invasion rate in MAC-T cells (cluster 5), could correspond to strains better adapted to the extracellular niche. We can hypothesized that strains 
of the first group, probably adapted to an intracellular niche, could lead to persistent infections by surviving inside the host cells, and that strains of the second group, probably adapted to an extracellular niche, could lead to acute infections, more easily eliminated by the host. All the intermediate cases (clusters 1, 3 and 4 ) could be related to a certain probability of chronicity.

To further challenge these correlations of chronic features, experiments will be now carried out on strains whose case histories are known and whose chronic tendency has been highlighted in vivo. Such confirmation could lead to the development of a predictive algorithmbased series of tests that would enable to predict the chronic tendency of a given strain and would thus help the farmer and/or the veterinarian to choose the best therapeutic approach subsequently to a mastitis diagnosis. Nevertheless, economical aspects of these kinds of tests have to be evaluated and compared to the currently taken measures in case of bovine mastitis.

\section{Acknowledgements}

This work was supported by Wallonia (SPW-DGARNE, convention D31-1231). We thank GSK Biologicals (Belgium) for the kind gift of the capsule antibodies.

\section{References}

Almeida, R.A., Matthews, K.R., Cifrian, E., Guidry, A.J., Oliver, S.P., 1996. Staphylococcus aureus invasion of bovine mammary epithelial cells. J. Dairy Sci. 79, 1021-1026.

Boulanger, D., Brouillette, E., Jaspar, F., Malouin, F., Mainil, J., Bureau, F., Lekeux, P., 2007. Helenalin reduces Staphylococcus aureus infection in vitro and in vivo. Vet. Microbiol. 119, 330-338.

Boulanger, D., Bureau, F., Melotte, D., Mainil, J., Lekeux, P., 2003. Increased nuclear factor kappaB activity in milk cells of mastitis-affected cows. J. Dairy Sci. 86, 1259-1267.

Brouillette, E., Grondin, G., Shkreta, L., Lacasse, P., Talbot, B.G., 2003. In vivo and in vitro demonstration that Staphylococcus aureus is an intracellular pathogen in the presence or absence of fibronectinbinding proteins. Microb. Pathogenesis 35, 159-168.

Buzzola, F.R., Alvarez, L.P., Tuchscherr, L.P., Barbagelata, M.S., Lattar, S.M., Calvinho, L., Sordelli, D.O., 2007. Differential abilities of capsulated and noncapsulated Staphylococcus aureus isolates from diverse agr groups to invade mammary epithelial cells. Infect. Immun. 75, 886-891.
Costerton, J.W., Stewart, P.S., Greenberg, E.P., 1999. Bacterial biofilms: a common cause of persistent infections. Science 284, 1318-1322.

Garzoni, C., Kelley, W.L., 2009. Staphylococcus aureus: new evidence for intracellular persistence. Trends Microbiol. 17, 59-65.

Gilot, P., Lina, G., Cochard, T., Poutrel, B., 2002. Analysis of the genetic variability of genes encoding the RNA III-activating components Agr and TRAP in a population of Staphylococcus aureus strains isolated from cows with mastitis. J. Clin. Microbiol. 40, 4060-4067.

Halasa, T., Huijps, K., Osteras, O., Hogeveen, H., 2007. Economic effects of bovine mastitis and mastitis management: a review. Vet. Q. 29, 18-31.

Kerro Dego, O., van Dijk, J.E., Nederbragt, H., 2002. Factors involved in the early pathogenesis of bovine Staphylococcus aureus mastitis with emphasis on bacterial adhesion and invasion. A review. Vet. Q. 24, 181-198.

Melchior, M.B., Vaarkamp, H., Fink-Gremmels, J., 2006. Biofilms: a role in recurrent mastitis infections? Vet. J. 171, 398-407.

Melchior, M.B., van Osch, M.H., Graat, R.M., van Duijkeren, E., Mevius, D.J., Nielen, M., Gaastra, W., Fink-Gremmels, J., 2009. Biofilm formation and genotyping of Staphylococcus aureus bovine mastitis isolates: evidence for lack of penicillin-resistance in Agr-type II strains. Vet. Microbiol. 137, 83-89.

Mitchell, G., Brouillette, E., Seguin, D.L., Asselin, A.E., Jacob, C.L., Malouin, F., 2010. A role for sigma factor B in the emergence of Staphylococcus aureus small-colony variants and elevated biofilm production resulting from an exposure to aminoglycosides. Microb. Pathogenesis 48, $18-27$.

Ote, I., Taminiau, B., Duprez, J.N., Dizier, I., Mainil, J.G., 2011. Genotypic characterization by polymerase chain reaction of Staphylococcus aureus isolates associated with bovine mastitis. Vet. Microbiol. 153, 285-292.

Pohlmann-Dietze, P., Ulrich, M., Kiser, K.B., Doring, G., Lee, J.C., Fournier, J.M., Botzenhart, K., Wolz, C., 2000. Adherence of Staphylococcus aureus to endothelial cells: influence of capsular polysaccharide, global regulator agr, and bacterial growth phase. Infect. Immun. 68, 4865-4871.

Stepanovic, S., Vukovic, D., Hola, V., Di Bonaventura, G., Djukic, S., Cirkovic, I., Ruzicka, F., 2007. Quantification of biofilm in microtiter plates: overview of testing conditions and practical recommendations for assessment of biofilm production by staphylococci. APMIS 115 , 891-899.

Tuchscherr, L., Loffler, B., Buzzola, F.R., Sordelli, D.O., 2010. Staphylococcus aureus adaptation to the host and persistence: role of loss of capsular polysaccharide expression. Future Microbiol. 5, 1823-1832.

Tuchscherr, L.P., Buzzola, F.R., Alvarez, L.P., Caccuri, R.L., Lee, J.C., Sordelli, D.O., 2005. Capsule-negative Staphylococcus aureus induces chronic experimental mastitis in mice. Infect. Immun. 73, 7932-7937.

Varhimo, E., Varmanen, P., Fallarero, A., Skogman, M., Pyorala, S. Iivanainen, A., Sukura, A., Vuorela, P., Savijoki, K., 2011. Alphaand beta-casein components of host milk induce biofilm formation in the mastitis bacterium Streptococcus uberis. Vet. Microbiol. 149, 381-389.

Watts, J.L., 1988. Etiological agents of bovine mastitis. Vet. Microbiol. 16, 41-66. 